\title{
Assessment of In-Place Oil Shale Resources of the Green River Formation, Piceance Basin, Western Colorado
}

Using a geology-based assessment methodology, the U.S. Geological Survey estimated a total of 1.525 trillion barrels of oil in place in seventeen oil shale zones in the Eocene Green River Formation in the Piceance Basin, western Colorado.

\section{Introduction}

The U.S. Geological Survey (USGS) recently completed a reassessment of in-place oil shale resources, regardless of richness, in the Eocene Green River Formation in the Piceance Basin, western Colorado (fig. 1). A considerable amount of oil-yield data has been collected after previous in-place assessments were published (Pitman and Johnson, 1978; Pitman, 1979; Pitman and others, 1989), and these data were incorporated into this new assessment. About twice as many oil-yield data points were used, and several additional oil shale intervals were included that were not assessed previously for lack of data. Oil yields are measured using the Fischer assay method. The Fischer assay method is a standardized laboratory test for determining the oil yield from oil shale that has been almost universally used to determine oil yields for Green River Formation oil shales (Stanfield and Frost, 1949; ASTM, 1980). Fischer assay does not necessarily measure the maximum amount of oil that an oil shale can produce, and there are retorting methods that yield more than the Fischer assay yield. However, the oil yields achieved by other technologies are typically reported as a percentage of the Fischer assay oil yield, and thus Fischer assay is still considered the standard by which other methods are compared.

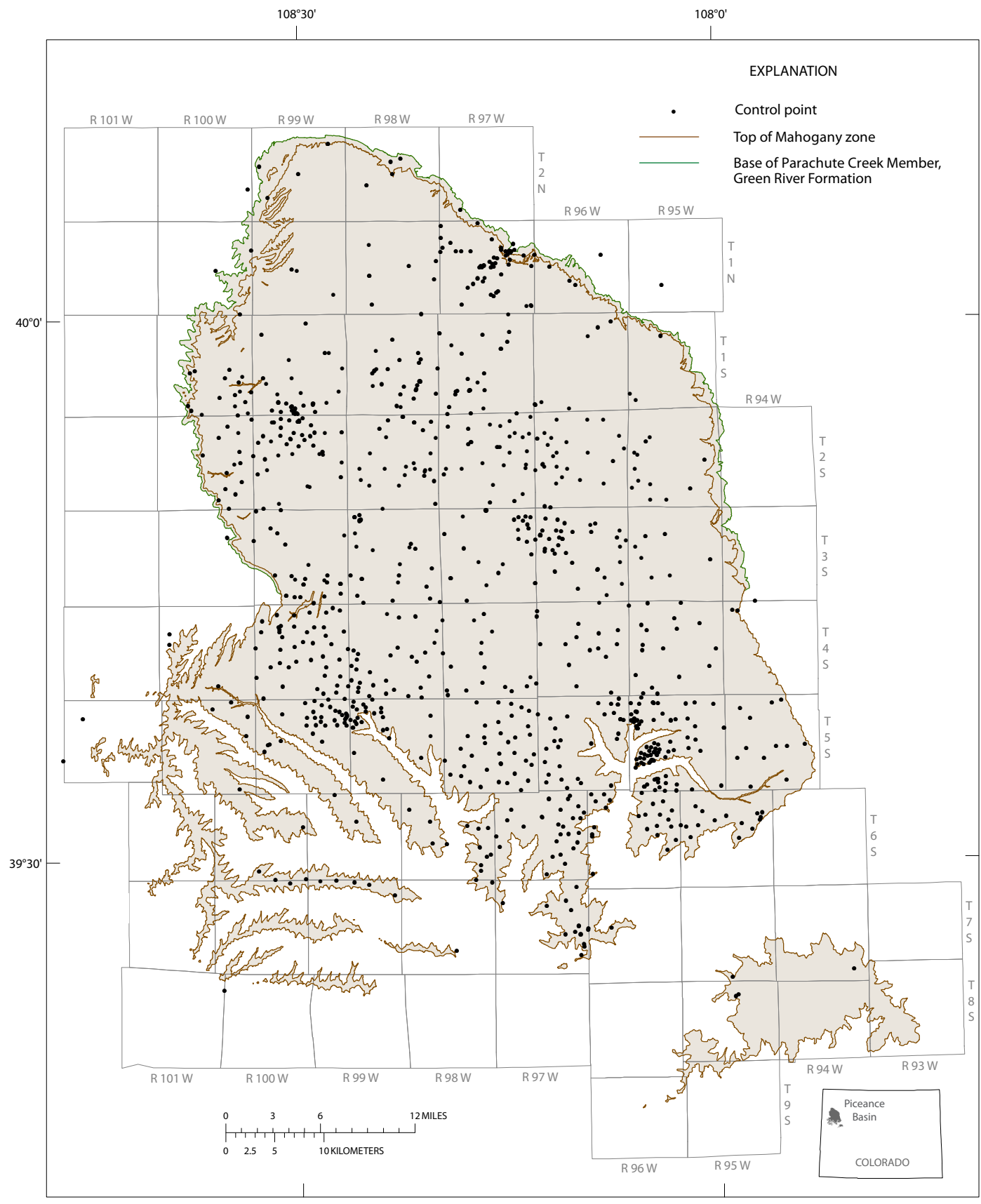

Figure 1. Map showing distribution of oil shale deposits in the Eocene Green River Formation, Piceance Basin, Colorado. Control points used in resource assessment are shown. 


\section{Methodology}

The oil shale interval in the Piceance Basin is subdivided into seventeen "rich" and "lean" zones (fig. 2) that were assessed separately. These zones, originally defined by Cashion and Donnell (1972), are roughly time-stratigraphic units consisting of distinctive, laterally continuous sequences of rich and lean oil shale beds that can be traced throughout much of the Piceance Basin. In this assessment, a spatial interpolation and extrapolation method for generating resource maps and computing resource volumes was used - the Radial Basis Function (RBF) in ArcGIS GeoStatistical Analyst (Environmental Systems Research Institute, Inc. (ESRI), Redlands, Calif., 2006, version 9.2).

The RBF method in GeoStatistical Analyst is an exact interpolator; it will honor all data points without introducing errors at those locations. Where it is important for the modeling method to honor the measured values, RBF can also extrapolate values above or below the actual values away from the data-point locations. The final resource models for each oil shale zone were created using a sampling method containing ten moving window sectors with eight neighbors in each sector.

Although the RBF method does not generate as complete an error estimate as kriging, it does give the difference between the predicted and measured value for each control point, and it does give an overall difference value for an entire oil shale zone model. To obtain the difference between the predicted value and the measured value, the RBF method predicts a value at a given control point from the nearest control points (in this assessment, eight neighbors are used) without knowing the actual value measured at that control point. That predicted value is then compared with the measured value, and the difference between the two is calculated.

\section{Resource Summary}

Table 1 lists the estimated total in-place oil for each oil shale zone in each township $\left(36 \mathrm{mi}^{2}\right)$ in the Piceance Basin. An "NA" on the resource table means that a particular zone was not assessed in that township due either to not being present or because of inadequate control. Richest oil shale zones in
Stratigraphic nomenclature for oil shale zones from Donnell and Blair (1970), Cashion and Donnell (1972)

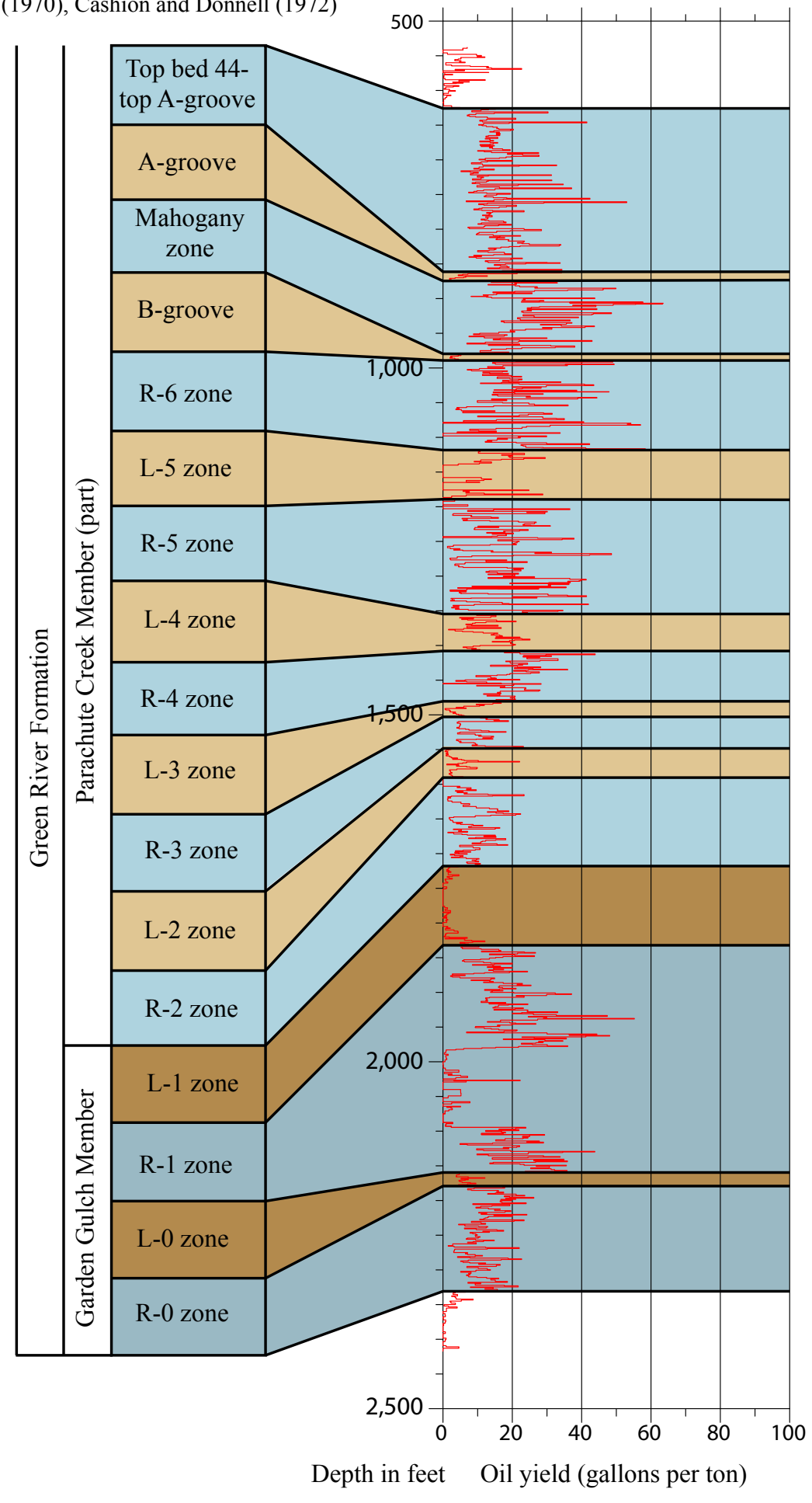

EXPLANATION

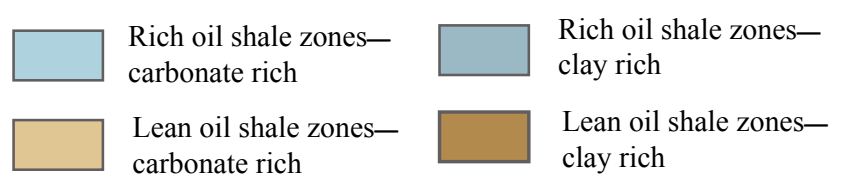

Figure 2. Chart showing oil yield in gallons per ton and the rich and lean oil shale zones in the Green River Formation assessed in this study. 

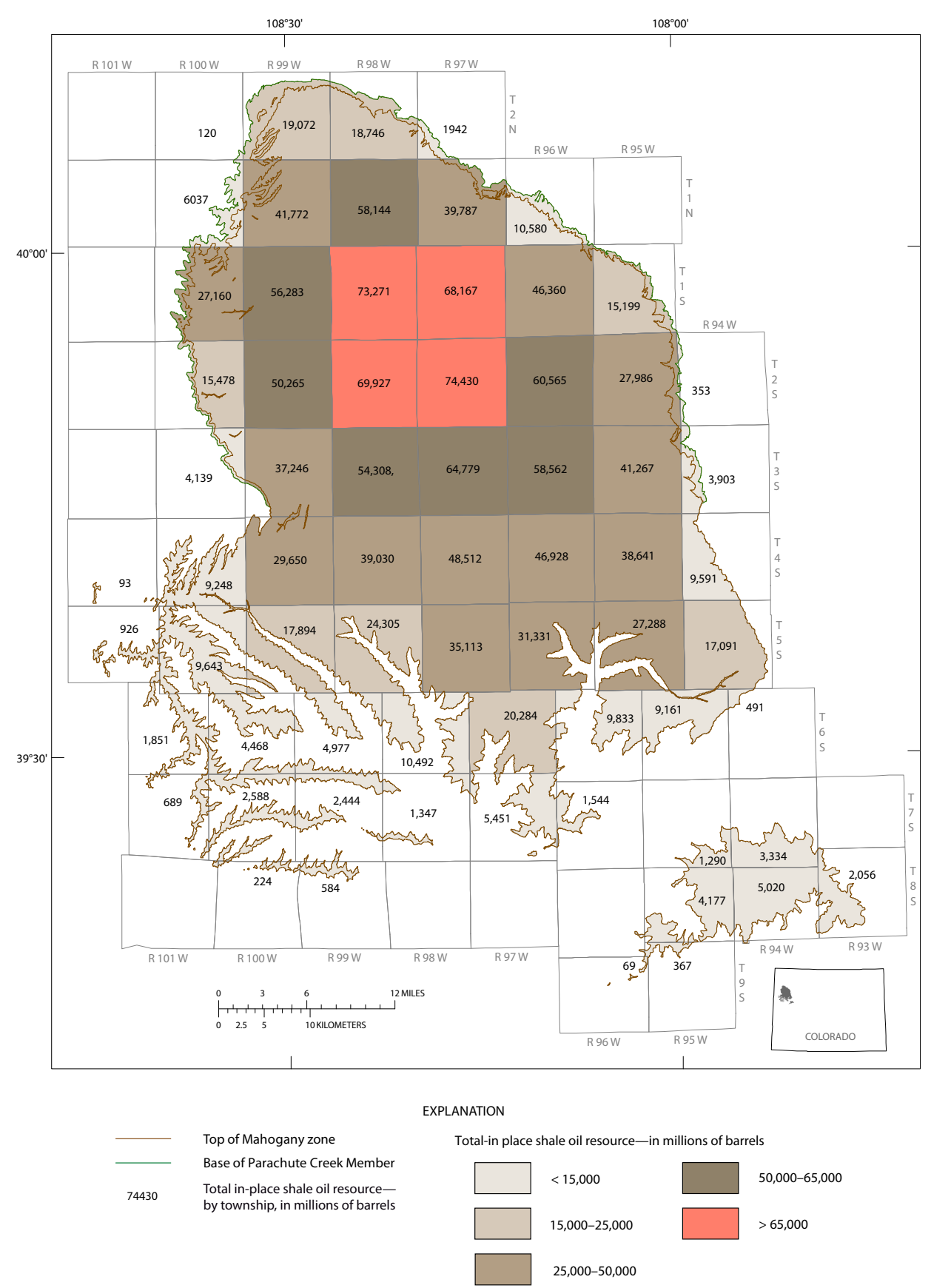

Figure 3. Map showing total in-place oil in millions of barrels as assessed for each township in the Piceance Basin, Colorado.

the basin are, in ascending stratigraphic order, (1) R-1 zone, 195.4 billion barrels; (2) R-5 zone, 198.2 billion barrels;

(3) R-6 zone, 185.4 billion barrels;

(4) Mahogany zone, 191.7 billion barrels; and (5) interval from Bed 44 to A-groove with 189.7 billion barrels. Total in-place oil in the seventeen oil shale zones assessed is 1.525 trillion barrels (table 1) or about 50 percent larger than the previous in-place assessment of about one trillion barrels. Almost all of this increase is due to (1) new areas being assessed that had too little data to assess in the previous assessment, and (2) new intervals being assessed that were not assessed previously. Much of this previously unassessed resource is of low grade. Figure 3 shows the total oil in place for all oil shale zones combined in each township in the basin. The richest resources are in Tps. 1 and 2 S., Rs. 97 and $98 \mathrm{~W}$. with a combined total of nearly 286 billion barrels of oil in place.

\section{References}

American Society for Testing and Materials (ASTM), 1980, Standard method of test for oil from oil shale: Annual Book of ASTM Standards, Part 25, Designation D 3904-80, p. 513-515.

Cashion, W.B., and Donnell, J.R., 1972, Chart showing correlation of selected key units in the organic-rich sequence of the Green River Formation, Piceance Creek basin, Colorado, and Uinta Basin, Utah: U.S. Geological Survey Oil and Gas Investigations Chart OC-65.

Donnell, J.R., and Blair, R.W., 1970, Resource appraisal of three rich oilshale zones in the Green River Formation, Piceance Creek basin, Colorado: Colorado School of Mines Quarterly, v. 65, p. $73-87$.

Pitman, J.K., 1979, Isopach, structure contour, and resource maps of the R-6 oil shale zone, Green River Formation, Piceance Creek basin, Colorado: U.S. Geological Survey Miscellaneous Field Investigations Map MF-1069, scale $1: 126,720$.

Pitman, J.K., and Johnson, R.C., 1978, Isopach, structure contour, and resource maps of the Mahogany oilshale zone, Green River Formation, Piceance Creek basin, Colorado: U.S. Geological Survey Miscellaneous Field Investigations Map MF-958, scale $1: 126,720$.

Pitman, J.K., Pierce, F.W., and Grundy, W.D., 1989, Thickness, oil-yield, and kriged resource estimates for the Eocene Green River Formation, Piceance Creek basin, Colorado: U.S. Geological Survey Oil and Gas Investigations Chart OC-132.

Stanfield, K.E., and Frost, I.C., 1949, Method of assaying oil shale by a modified Fischer retort: U.S. Bureau of Mines Report of Investigations 4477. 
Table 1. Total in-place shale oil resources by township and oil shale zone in the Parachute Creek Member of the Green River Formation, Piceance Basin, Garfield and Rio Blanco Counties, northwest Colorado. Resource values are shown in millions of barrels of shale oil.

1 barrel of oil $=42$ gallons.

[All values calculated by ESRI's ArcGIS GeoStatistical Analyst extension with a Radial Basis Function (RBF) Multiquadratic Gridding Function; NA, not assessed]

\begin{tabular}{|c|c|c|c|c|c|c|c|c|c|c|}
\hline Township and Range & 1N. 96W. & 1N. 97W. & 1N. 98W. & 1N. 99W. & 1N. 100W. & 2N. 97W. & 2N. 98W. & 2N. 99W. & 2N.100W. & 1S. 95W. \\
\hline R-0 zone & 90.71 & $1,309.35$ & $3,437.06$ & $2,961.75$ & 493.07 & 153.73 & $1,577.62$ & $1,737.70$ & 10.66 & 458.31 \\
\hline L-0 zone & 78.62 & 291.73 & 347.06 & 223.74 & 37.09 & 21.48 & 137.50 & 131.64 & 0.85 & 81.79 \\
\hline $\mathrm{R}-1$ & $1,474.42$ & $5,233.07$ & $6,010.24$ & $5,711.78$ & $1,198.22$ & 235.43 & $2,590.34$ & $3,194.78$ & 26.34 & $1,644.14$ \\
\hline L-1 & 88.66 & 413.01 & 401.78 & 400.10 & 82.38 & 23.06 & 181.75 & 223.72 & 1.67 & 253.50 \\
\hline R-2 & 531.28 & $1,995.89$ & $3,041.92$ & $2,393.75$ & 460.87 & 145.58 & $1,350.17$ & $1,502.56$ & 10.93 & 941.00 \\
\hline L-2 & 173.20 & 657.99 & $1,306.47$ & 933.54 & 142.62 & 47.13 & 532.77 & 560.72 & 3.70 & 469.14 \\
\hline R-3 & 467.19 & $2,301.64$ & $3,891.74$ & $3,106.60$ & 482.95 & 119.14 & $1,321.60$ & $1,612.73$ & 12.13 & 698.68 \\
\hline L-3 & 204.32 & 726.38 & $1,317.69$ & 820.37 & 118.69 & 35.92 & 435.71 & 406.37 & 2.54 & 313.86 \\
\hline $\mathrm{R}-4$ & $1,155.68$ & $4,241.89$ & $6,682.88$ & $5,121.74$ & 782.50 & 243.59 & $1,841.36$ & $2,168.90$ & 17.86 & $1,861.57$ \\
\hline L-4 & 590.49 & $2,634.00$ & $4,156.20$ & $2,614.72$ & 400.65 & 100.60 & 818.33 & 846.96 & 7.37 & 918.81 \\
\hline R-5 & $1,867.63$ & $6,313.40$ & $9,373.94$ & $6,041.55$ & 980.27 & 321.64 & $2,489.14$ & $2,358.30$ & 18.03 & $2,711.60$ \\
\hline L-5 & 634.13 & $2,000.49$ & $3,276.53$ & $2,060.38$ & 369.63 & 96.24 & $1,163.47$ & $1,250.38$ & 8.29 & $1,196.01$ \\
\hline R-6 & $1,612.99$ & $5,764.13$ & $7,794.81$ & $5,052.84$ & 260.56 & 216.10 & $2,503.07$ & $1,616.96$ & NA & $1,762.02$ \\
\hline B-groove & 78.29 & 219.57 & 231.56 & 136.24 & 6.14 & 6.85 & 82.27 & 63.10 & NA & 81.41 \\
\hline Mahogany & $1,505.32$ & $5,581.22$ & $6,700.01$ & $4,031.95$ & 212.84 & 171.40 & $1,639.46$ & $1,311.13$ & NA & $1,688.44$ \\
\hline A-Groove & 27.33 & 102.76 & 173.95 & 160.76 & 8.49 & 3.88 & 81.50 & 86.04 & NA & 118.61 \\
\hline Bed 44-A-groove & NA & NA & NA & NA & NA & NA & NA & NA & NA & NA \\
\hline
\end{tabular}

\begin{tabular}{|c|c|c|c|c|c|c|c|c|c|c|}
\hline Township and Range & 1S. 96W. & 1S. $97 W$. & 1S. 98W. & 1S. 99W. & 1S. 100W. & 2S. 94W. & 2S. 95W. & 2S. 96W. & 2S. 97W. & 2S. 98W. \\
\hline $\mathrm{R}-0$ zone & $1,306.03$ & $2,895.85$ & $4,807.90$ & $3,977.93$ & $1,964.36$ & 15.91 & $1,286.65$ & $2,467.07$ & $3,570.45$ & $4,323.48$ \\
\hline L-0 zone & 271.22 & 448.85 & 460.33 & 286.16 & 162.63 & 2.42 & 178.49 & 358.50 & 508.63 & 354.29 \\
\hline R-1 & $5,125.46$ & $6,068.66$ & $5,327.17$ & $5,697.93$ & $3,998.57$ & 64.98 & $4,416.89$ & $6,331 \cdot 14$ & $6,012.89$ & $5,684.98$ \\
\hline L-1 & 651.28 & 631.33 & 512.15 & 591.72 & 376.60 & 7.81 & 452.88 & 677.64 & 582.45 & 562.62 \\
\hline $\mathrm{R}-2$ & $2,296.15$ & $3,562.77$ & $4,017.47$ & $2,851.52$ & $1,637.28$ & 29.54 & $1,827.29$ & $3,093.13$ & $3,778.05$ & $3,376.42$ \\
\hline L-2 & $1,192.26$ & $1,539.67$ & $1,504.35$ & $1,185.05$ & 473.64 & 12.73 & 769.42 & $1,315.28$ & $1,420.65$ & $1,411.00$ \\
\hline R-3 & $2,552.27$ & $5,236.09$ & $6,462.24$ & $4,329.57$ & $1,176.29$ & 16.47 & $1,289.44$ & $2,999.68$ & $4,813.80$ & $5,177.65$ \\
\hline L-3 & 954.77 & $1,396.08$ & $1,415.69$ & $1,145.20$ & 344.37 & 8.93 & 517.76 & $1,031.55$ & $1,569.08$ & $1,287.36$ \\
\hline R-4 & $5,192.26$ & $7,977.04$ & $8,922.47$ & $6,739.63$ & $2,046.91$ & 52.05 & $2,936.51$ & $5,327.00$ & $7,722.11$ & $6,879.68$ \\
\hline L-4 & $2,862.56$ & $5,220.36$ & $6,087.86$ & $3,809.77$ & $1,241.66$ & 26.04 & $1,567.83$ & $2,792.61$ & $4,003.38$ & $4,406.57$ \\
\hline R-5 & $7,548.34$ & $12,056.60$ & $13,357.50$ & $10,141.90$ & $3,471.16$ & 76.57 & $4,128.26$ & $7,192.46$ & $11,062.40$ & $11,177.00$ \\
\hline L-5 & $2,672.97$ & $3,238.02$ & $4,023.31$ & $2,741.84$ & $1,262.56$ & 39.50 & $2,062.85$ & $2,840.82$ & $3,204.27$ & $2,691.17$ \\
\hline R-6 & $6,580.23$ & $8,520.93$ & $7,849.06$ & $6,226.68$ & $2,998.86$ & NA & $3,038.56$ & $6,985.57$ & $7,655.86$ & $6,617.41$ \\
\hline B-groove & 300.18 & 361.61 & 299.04 & 244.49 & 104.70 & NA & 99.32 & 195.26 & 233.28 & 281.61 \\
\hline Mahogany & $6,549.13$ & $8,747.78$ & $8,034.57$ & $6,151.92$ & $2,581.55$ & NA & $3,219.13$ & $6,843.01$ & $7,983.07$ & $7,124.94$ \\
\hline A-Groove & 305.33 & 265.20 & 189.83 & 161.46 & 89.71 & NA & 194.31 & 281.24 & 228.80 & 139.03 \\
\hline Bed 44-A-groove & NA & NA & NA & NA & NA & NA & NA & $9,832.81$ & $10,081.00$ & $8,432.09$ \\
\hline
\end{tabular}

\begin{tabular}{|c|c|c|c|c|c|c|c|c|c|c|}
\hline Township and Range & 2S. 99W. & 2S. 100W. & 3S. $94 W$. & 3S. 95W. & 3S. $96 W$. & 3S. 97W. & 3S. 98W. & 3S. 99W. & 3S. 100W. & 4S. 94W. \\
\hline R-0 zone & $3,508.29$ & $1,353.83$ & 78.65 & $1,438.66$ & $2,936.60$ & $3,548.70$ & $3,696.50$ & $2,960.79$ & 374.62 & 91.13 \\
\hline L-0 zone & 296.48 & 122.88 & 12.59 & 158.24 & 287.41 & 398.76 & 304.33 & 234.56 & 31.06 & 16.07 \\
\hline $\mathrm{R}-1$ & $5,924.60$ & $3,120.14$ & 331.40 & $5,038.42$ & $7,690.24$ & $7,209.92$ & $7,159.30$ & $6,438.08$ & 906.36 & 419.32 \\
\hline L-1 & 430.51 & 202.11 & 27.93 & 355.33 & 691.40 & 876.16 & 719.79 & 456.45 & 69.40 & 16.08 \\
\hline $\mathrm{R}-2$ & $2,624.66$ & 936.63 & 153.48 & $1,476.81$ & $3,139.00$ & $3,900.50$ & $3,443.69$ & $1,664.15$ & 140.58 & 181.71 \\
\hline L-2 & $1,007.59$ & 248.45 & 58.88 & 480.72 & 934.41 & $1,281.79$ & $1,160.34$ & 560.45 & 31.65 & 67.75 \\
\hline R-3 & $3,004.47$ & 470.62 & 87.63 & 854.42 & $2,034.62$ & $3,263.15$ & $2,376.52$ & $1,072.22$ & 57.03 & 119.55 \\
\hline L-3 & 744.93 & 141.34 & 59.38 & 457.00 & 798.27 & $1,008.67$ & 682.46 & 301.68 & 13.97 & 103.14 \\
\hline R-4 & $4,122.51$ & $1,183.92$ & 341.07 & $2,439.39$ & $3,879.44$ & $4,893.66$ & $3,948.71$ & $2,226.10$ & 230.26 & 603.96 \\
\hline L-4 & $2,192.05$ & 623.33 & 168.59 & $1,289.40$ & $2,056.73$ & $2,586.68$ & $2,053.78$ & $1,252.79$ & 115.55 & 297.37 \\
\hline R-5 & $7,090.78$ & $2,112.34$ & 571.96 & $3,927.01$ & $6,253.97$ & $7,446.49$ & $6,624.39$ & $4,180.00$ & 431.86 & $1,173.31$ \\
\hline L-5 & $1,594.80$ & 663.53 & 246.79 & $1,827.50$ & $2,374.11$ & $2,423.42$ & $1,693.74$ & $1,059.83$ & 104.85 & 428.26 \\
\hline R-6 & $4,854.46$ & $2,163.16$ & 433.60 & $5,927.05$ & $7,757.86$ & $7,376.38$ & $5,840.64$ & $3,931.63$ & 439.85 & $1,499.46$ \\
\hline B-groove & 172.29 & 106.63 & 16.68 & 180.35 & 256.22 & 459.13 & 177.12 & 173.32 & 34.34 & 75.70 \\
\hline Mahogany & $5,258.36$ & $1,965.48$ & 401.10 & $6,222.47$ & $7,545.47$ & $7,966.01$ & $6,058.93$ & $4,157.58$ & 436.36 & $1,465.50$ \\
\hline A-Groove & 140.26 & 63.97 & 18.36 & 180.31 & 203.94 & 176.09 & 178.40 & 180.86 & 15.66 & 98.85 \\
\hline Bed 44-A-groove & 7,297.94 & NA & 895.04 & $9,014.21$ & $9,721.99$ & $9,963.75$ & $8,189.66$ & $6,395.67$ & NA & $2,933.40$ \\
\hline
\end{tabular}


Table 1. Total in-place shale oil resources by township and oil shale zone in the Parachute Creek Member of the Green River Formation, Piceance Basin, Garfield and Rio Blanco Counties, northwest Colorado. Resource values are shown in millions of barrels of shale oil.

1 barrel of oil $=42$ gallons. - Continued

[All values calculated by ESRI's ArcGIS GeoStatistical Analyst extension with a Radial Basis Function (RBF) Multiquadratic Gridding Function; NA, not assessed]

\begin{tabular}{|c|c|c|c|c|c|c|c|c|c|c|}
\hline Township and Range & 4S. 95W. & 4S. 96W. & 4S. $97 \mathrm{~W}$. & 4S. 98W. & 4S. 99W. & 4S. 100W. & 4S. 101W. & 5S. 94W. & 5S. 95W. & 5S. 96W. \\
\hline R-0 zone & $1,190.95$ & $2,449.07$ & $3,270.71$ & $2,739.03$ & $2,268.90$ & 731.13 & 4.83 & 143.81 & 783.69 & $1,523.76$ \\
\hline L-0 zone & 109.66 & 207.75 & 265.96 & 218.25 & 157.91 & 56.81 & 0.61 & 24.26 & 69.85 & 126.11 \\
\hline R-1 & $3,986.71$ & $7,468.62$ & $9,439.64$ & $8,031.78$ & $6,319.22$ & $2,249.36$ & 14.81 & 592.64 & $2,606.03$ & $4,985.71$ \\
\hline L-1 & 185.07 & 415.14 & 635.22 & 591.38 & 381.29 & 135.20 & 1.06 & 11.85 & 56.93 & 173.12 \\
\hline R-2 & 915.45 & $1,589.77$ & $1,945.46$ & $1,436.89$ & 781.39 & 113.45 & 0.01 & 155.57 & 306.58 & 616.89 \\
\hline L-2 & 268.01 & 376.91 & 549.81 & 283.21 & 144.60 & 24.02 & 0.03 & 87.70 & 145.78 & 190.24 \\
\hline R-3 & 576.41 & 897.00 & $1,030.74$ & 644.40 & 401.71 & 76.45 & 0.04 & 170.80 & 326.16 & 453.05 \\
\hline L-3 & 349.91 & 420.61 & 453.61 & 369.39 & 324.22 & 70.77 & 0.15 & 168.44 & 232.84 & 266.08 \\
\hline $\mathrm{R}-4$ & $2,110.90$ & $2,695.35$ & $2,708.38$ & $2,241.35$ & $1,551.64$ & 494.13 & 3.50 & $1,051.72$ & $1,459.02$ & $1,700.24$ \\
\hline L-4 & $1,144.45$ & $1,427.76$ & $1,528.54$ & $1,022.19$ & 760.93 & 230.22 & 1.36 & 463.95 & 742.96 & 813.06 \\
\hline R-5 & $4,214.38$ & $4,964.98$ & $4,845.95$ & $3,960.32$ & $2,827.29$ & $1,004.77$ & 10.23 & $1,802.37$ & $2,881.68$ & $3,243.39$ \\
\hline L-5 & $1,672.03$ & $2,031.25$ & $1,936.79$ & $1,426.39$ & 860.36 & 246.11 & 1.42 & 489.82 & $1,121.24$ & $1,412.85$ \\
\hline R-6 & $6,579.42$ & $6,915.16$ & $5,773.39$ & $4,227.30$ & $3,107.90$ & $1,207.48$ & 10.28 & $2,316.05$ & $4,782.84$ & $4,796.21$ \\
\hline B-groove & 205.57 & 190.14 & 180.43 & 134.39 & 110.21 & 63.21 & 0.31 & 202.15 & 180.20 & 158.64 \\
\hline Mahogany & $6,420.54$ & $6,535.60$ & $5,834.74$ & $4,538.40$ & $3,641.75$ & $1,391.08$ & 13.90 & $3,427.43$ & $4,944.05$ & $4,644.92$ \\
\hline A-Groove & 198.16 & 180.22 & 150.25 & 148.46 & 137.51 & 57.93 & 0.63 & 150.12 & 164.83 & 116.49 \\
\hline Bed 44-A-groove & $8,512.92$ & $8,162.23$ & $7,962.17$ & $7,017.09$ & $5,872.87$ & $2,303.53$ & 29.61 & $5,832.41$ & $6,483.44$ & $6,110.43$ \\
\hline Township and Range & 5S. $97 W$. & 5S. 98W. & 5S. 99W. & 5S. 100W. & 5S. 101W. & 6S. 94W. & 6S. 95W. & 6S. 96W. & 6S. 97W. & 6S. 98W. \\
\hline R-0 zone & $2,150.75$ & $1,558.55$ & $1,193.32$ & 666.57 & 57.91 & 1.73 & 184.51 & 345.85 & $1,056.05$ & 629.14 \\
\hline L-0 zone & 166.79 & 122.82 & 90.24 & 53.28 & 6.51 & 0.74 & 19.99 & 31.97 & 89.02 & 48.76 \\
\hline $\mathrm{R}-1$ & $7,082.52$ & $5,272.85$ & $3,777.32$ & $1,941.63$ & 132.59 & 6.94 & 576.91 & $1,111.15$ & $3,362.65$ & $2,004.86$ \\
\hline L-1 & 290.69 & 275.37 & 216.70 & 114.43 & 8.33 & 0.47 & 13.09 & 29.28 & 171.56 & 143.58 \\
\hline R-2 & 702.37 & 436.66 & 249.30 & 63.30 & 0.53 & 2.21 & 47.90 & 97.27 & 316.50 & 164.67 \\
\hline L-2 & 198.51 & 98.50 & 46.52 & 13.80 & 1.42 & 1.54 & 32.73 & 46.70 & 98.46 & 40.04 \\
\hline R-3 & 480.10 & 315.61 & 214.88 & 80.70 & 4.17 & 2.67 & 72.11 & 112.57 & 280.96 & 139.67 \\
\hline L-3 & 319.98 & 246.02 & 200.69 & 82.31 & 4.86 & 3.51 & 78.13 & 81.16 & 160.49 & 83.16 \\
\hline R-4 & $1,863.92$ & $1,283.57$ & 904.98 & 469.14 & 47.44 & 28.85 & 470.38 & 530.92 & $1,176.85$ & 567.46 \\
\hline L-4 & 869.18 & 514.17 & 356.79 & 190.02 & 19.66 & 8.95 & 160.69 & 199.34 & 367.25 & 195.92 \\
\hline R-5 & $3,538.30$ & $2,274.38$ & $1,629.68$ & 967.75 & 119.44 & 37.54 & 682.66 & 878.18 & $1,983.84$ & $1,048.54$ \\
\hline L-5 & $1,285.61$ & 869.30 & 579.08 & 245.10 & 17.13 & 13.12 & 288.17 & 374.98 & 738.65 & 313.68 \\
\hline R-6 & $4,096.45$ & $2,705.87$ & $2,060.04$ & $1,129.54$ & 103.18 & 75.15 & $1,454.55$ & $1,468.84$ & $2,311.66$ & $1,106.72$ \\
\hline B-groove & 167.32 & 141.40 & 83.44 & 47.04 & 3.33 & 6.50 & 135.56 & 56.10 & 100.90 & 62.27 \\
\hline Mahogany & $4,640.82$ & $3,082.83$ & $2,422.31$ & $1,288.56$ & 135.30 & 104.50 & $2,010.70$ & $1,919.93$ & $3,328.92$ & $1,631.06$ \\
\hline A-Groove & 133.57 & 98.79 & 90.31 & 67.24 & 7.94 & 4.11 & 71.48 & 45.46 & 87.81 & 49.26 \\
\hline Bed 44-A-groove & $7,126.06$ & $5,008.44$ & $3,778.77$ & $2,223.04$ & 256.33 & 192.60 & $2,861.28$ & $2,503.13$ & $4,652.46$ & $2,263.30$ \\
\hline Township and Range & 6S. 99W. & 6S. 100W. & 6S. 101W. & 7S. 93W. & 7S. 94W. & 7S. 95W. & 7S. 96W. & 7S. $97 \mathrm{~W}$. & 7S. 98W. & 7S. 99W. \\
\hline R-0 zone & 331.72 & 324.22 & 127.53 & NA & NA & 0.98 & 57.35 & 240.43 & 79.72 & 167.10 \\
\hline L-0 zone & 24.83 & 27.33 & 10.06 & NA & 2.02 & 2.25 & 5.67 & 23.18 & 6.46 & 12.92 \\
\hline $\mathrm{R}-1$ & $1,033.23$ & 842.07 & 259.20 & NA & NA & 5.49 & 170.29 & 702.02 & 208.36 & 431.75 \\
\hline L-1 & 67.50 & 52.27 & 17.11 & NA & NA & 0.35 & 5.38 & 27.46 & 15.85 & 32.23 \\
\hline $\mathrm{R}-2$ & 71.33 & 36.44 & 5.37 & NA & NA & 0.41 & 21.26 & 90.28 & 23.38 & 38.75 \\
\hline L-2 & 13.05 & 7.46 & 1.92 & NA & 2.22 & 1.86 & 6.00 & 22.31 & 4.70 & 5.69 \\
\hline R-3 & 76.40 & 61.36 & 15.26 & NA & 0.28 & 2.96 & 21.96 & 87.78 & 20.81 & 47.37 \\
\hline L-3 & 48.09 & 43.38 & 14.04 & NA & 3.01 & 3.52 & 9.71 & 34.68 & 8.94 & 19.28 \\
\hline R-4 & 249.84 & 216.19 & 90.76 & 0.08 & 160.74 & 60.73 & 90.02 & 335.77 & 79.17 & 120.98 \\
\hline L-4 & 73.91 & 64.29 & 35.53 & 0.01 & 23.88 & 7.99 & 11.63 & 41.34 & 11.35 & 13.02 \\
\hline R-5 & 436.44 & 415.46 & 206.62 & 0.06 & 134.50 & 55.77 & 118.59 & 462.31 & 122.81 & 188.51 \\
\hline L-5 & 137.73 & 103.65 & 33.19 & 0.03 & 67.44 & 30.71 & 44.17 & 153.38 & 32.01 & 43.00 \\
\hline R-6 & 533.44 & 503.57 & 219.28 & 0.27 & 584.06 & 221.55 & 200.09 & 658.07 & 152.07 & 255.79 \\
\hline $\mathrm{B}$-groove & 28.70 & 25.65 & 10.20 & 0.09 & 153.34 & 42.83 & 11.09 & 33.38 & 9.97 & 17.35 \\
\hline Mahogany & 755.48 & 655.30 & 279.00 & 0.56 & 704.19 & 303.46 & 323.31 & $1,058.71$ & 227.38 & 435.37 \\
\hline A-Groove & 23.81 & 36.41 & 19.02 & 0.04 & 59.87 & 14.21 & 9.19 & 33.48 & 14.51 & 26.37 \\
\hline Bed 44-A-groove & $1,071.83$ & $1,053.47$ & 507.00 & 1.28 & $1,438.67$ & 534.87 & 438.60 & $1,446.49$ & 329.64 & 588.19 \\
\hline
\end{tabular}


Table 1. Total in-place shale oil resources by township and oil shale zone in the Parachute Creek Member of the Green River Formation, Piceance Basin, Garfield and Rio Blanco Counties, northwest Colorado. Resource values are shown in millions of barrels of shale oil. 1 barrel of oil $=42$ gallons. - Continued

[All values calculated by ESRI's ArcGIS GeoStatistical Analyst extension with a Radial Basis Function (RBF) Multiquadratic Gridding Function; NA, not assessed]

\begin{tabular}{|c|c|c|c|c|c|c|c|c|c|c|}
\hline Township and Range & 7S. 100W. & 7S. 101W. & 8S. 93W. & 8S. 94W. & 8S. 95W. & 8S. $96 \mathrm{~W}$. & 8S. 99W. & 8S. 100W. & 8S. 101W. & 9S. 94W. \\
\hline R-0 zone & 197.84 & 50.86 & NA & NA & 0.60 & 0.02 & 36.74 & 16.17 & 1.76 & NA \\
\hline L-0 zone & 17.99 & 5.36 & NA & 2.70 & 6.66 & 0.09 & 2.84 & 1.49 & 0.20 & NA \\
\hline $\mathrm{R}-1$ & 336.29 & 58.70 & NA & NA & 5.10 & 0.20 & 53.15 & 16.53 & 0.58 & NA \\
\hline L-1 & 25.11 & 5.02 & NA & NA & 0.58 & 0.05 & 4.94 & 1.68 & 0.10 & NA \\
\hline $\mathrm{R}-2$ & 21.52 & 2.00 & NA & NA & 1.53 & 0.09 & 7.18 & 1.96 & 0.09 & NA \\
\hline $\mathrm{L}-2$ & 5.42 & 1.24 & 0.27 & 3.40 & 6.50 & 0.10 & 1.26 & 0.56 & 0.07 & NA \\
\hline $\mathrm{R}-3$ & 35.82 & 5.58 & NA & 0.03 & 8.33 & 0.17 & 8.54 & 2.68 & 0.12 & NA \\
\hline L-3 & 19.28 & 4.16 & NA & 1.69 & 8.85 & 0.12 & 3.24 & 1.20 & 0.10 & NA \\
\hline $\mathrm{R}-4$ & 92.92 & 24.05 & 83.82 & 201.04 & 161.28 & 1.18 & 21.91 & 5.54 & 0.24 & 0.33 \\
\hline L-4 & 17.73 & 9.45 & 13.08 & 24.52 & 17.33 & 0.12 & NA & 0.16 & 0.09 & 0.03 \\
\hline $\mathrm{R}-5$ & 206.45 & 74.18 & 61.91 & 149.61 & 128.43 & 0.94 & 37.37 & 14.73 & 1.81 & 0.18 \\
\hline L-5 & 33.56 & 6.97 & 27.05 & 87.16 & 79.02 & 0.60 & 4.65 & 0.70 & 0.01 & 0.14 \\
\hline $\mathrm{R}-6$ & 280.38 & 77.45 & 348.55 & 902.56 & 730.24 & 7.04 & 74.79 & 25.82 & 2.74 & 2.07 \\
\hline B-groove & 20.72 & 5.23 & 119.81 & 251.71 & 157.13 & 1.29 & 5.58 & 2.47 & 0.20 & 0.52 \\
\hline Mahogany & 522.75 & 136.93 & 405.64 & $1,072.02$ & $1,009.07$ & 11.01 & 130.34 & 54.14 & 5.34 & 2.25 \\
\hline A-Groove & 30.20 & 8.61 & 45.04 & 79.98 & 28.90 & 0.28 & 10.06 & 3.89 & 0.40 & 0.12 \\
\hline Bed 44-A-groove & 724.33 & 212.91 & 950.54 & $2,243.21$ & $1,827.53$ & 18.65 & 181.76 & 74.26 & 8.16 & 4.53 \\
\hline
\end{tabular}

\begin{tabular}{lrrc}
\hline Township and Range & 9S. 95W. & 9S. 96W. & Grand Total By Township: \\
\hline R-0 zone & NA & NA & 83,400 \\
L-0 zone & 0.64 & 0.14 & 8,300 \\
R-1 & NA & NA & 195,400 \\
L-1 & 0.14 & 0.06 & 15,100 \\
R-2 & 0.16 & 0.01 & 66,800 \\
L-2 & 0.66 & 0.14 & 24,200 \\
R-3 & 0.75 & 0.13 & 68,100 \\
L-3 & 0.81 & 0.15 & 22,500 \\
R-4 & 9.95 & 1.27 & 127,200 \\
L-4 & 0.93 & 0.12 & 69,100 \\
R-5 & 6.66 & 0.65 & 198,200 \\
L-5 & 5.21 & 0.67 & 66,100 \\
R-6 & 65.22 & 12.21 & 185,400 \\
B-groove & 13.50 & 2.50 & 7,800 \\
Mahogany & 94.43 & 18.55 & 6,300 \\
A-Groove & 2.61 & 0.59 & 189,700 \\
Bed 44-A-groove & 164.85 & 31.84 & $\mathbf{1 , 5 2 5 , 2 0 0}$ \\
\hline Piceance Basin Grand Total & & & \\
\hline
\end{tabular}

\section{For Additional Information}

Supporting geologic studies of the oil shale-bearing units, assessment units, oil shale analysis, and the methodology used in assessing the oil shale resources in the Piceance Basin are in progress. Assessment results are available at the USGS Central Energy Team website: http://energy.cr.usgs.gov/other/oil_shale/.

\section{Contact Information}

For further information contact Ronald C. Johnson (rcjohnson@usgs.gov),T.J. Mercier(tmercier@usgs.gov), Michael E. Brownfield (mbrownfield@usgs.gov), Michael P. Pantea (mpantea@usgs.gov), or Jesse G. Self (jself@usgs.gov). 